\title{
RISK FACTORS FOR DEVELOPMENT OF MULTIDRUG-RESISTANT TUBERCULOSIS AMONG RELAPSED PATIENTS IN WEST PAPUA, INDONESIA: A DESCRIPTIVE AND ANALYTICAL STUDY
}

\author{
MOHAMMAD A. WIJAYANTO*, RAIHAN A. ARNANDA, EDO P. THAMRIN \\ Sorong Regency Regional General Hospital, West Papua, Indonesia \\ Email: mohammad.anom@gmail.com
}

Received: 15 Dec 2018, Revised and Accepted: 10 Mar 2019

\begin{abstract}
Objective: Indonesia has one of the highest burdens of multidrug-resistant tuberculosis (MDR-TB), with 6800 new cases of MDR-TB annually and a previously reported cure rate of 51\%. This study aimed to identify the risk factors that affect the development of MDR-TB in the diverse population of West Papua.
\end{abstract}

Methods: A case-control study was conducted in Sorong Regency Regional Hospital, a referral center for MDR-TB in West Papua. Data were obtained from medical records of patients with relapsed TB between January 2014 and September 2017. Extracted data included demographic characteristics, family history, medication history (type of medication and duration), smoking history, supporting examinations (sputum test for acid-fast bacilli [AFB] and radiologist interpretation of thoracic X-ray), and comorbidities (HIV and diabetes mellitus).

Results: Among 549 patients with suspected MDR-TB, 45 were confirmed to have MDR-TB. These 45 patients were defined as the case group and compared with a control group of 45 relapsed patients who had drug-sensitive TB. A great number of subjects in both case and control groups $(44.4 \%$ and $57.8 \%)$ were categorized as previously lost to follow-up. Bivariate analysis using the chi-squared test found that an AFB result of +3 (odds ratio [OR]: 5.33, 95\% confidence interval [CI] 1.76-16.09), diabetes mellitus (OR: 6.14, 95\% CI 1.26-29.89), and completion of intensivephase category I anti-TB therapy (OR: 3.25, 95\% CI 1.04-10.07) were associated with MDR-TB.

Conclusion: These clinical variables provide initial information about MDR-TB in West Papua and will assist clinicians to manage patients in related populations who have these risk factors.

Keywords: Multidrug-resistant tuberculosis, Indonesia, West Papua

(C) 2019 The Authors. Published by Innovare Academic Sciences Pvt Ltd. This is an open access article under the CC BY license (http://creativecommons. org/licenses/by/4. 0/) DOI: http://dx.doi.org/10.22159/ijap.2019.v11s6.33539

\section{INTRODUCTION}

The magnitude of the global problem of tuberculosis (TB) disease is relatively high but differs around the world. TB is regarded as one of the top 10 leading causes of death worldwide and the leading cause of death from a single infectious disease. According to the latest World Health Organization (WHO) global report in 2017, 10.4 million people were estimated to be ill from TB, with an estimated 1.3 million deaths among HIV-negative TB patients and an additional 374,000 deaths among HIV-positive TB patients. Most cases in 2016 occurred in Asia, with $56 \%$ of total cases estimated to occur in India, Indonesia, China, Philippines, and Pakistan [1].

The development of drug-resistant TB during treatment remains the main problem. Global WHO surveillance in 2017 reported 490,000 cases of multidrug-resistant TB (MDR-TB) and 110,000 cases of rifampicin-resistant TB (RR-TB) in 2016. From another perspective, cases of MDR-TB represent $4.1 \%$ of newly diagnosed cases and $19 \%$ of cases of relapsed previously treated TB [1-4].

According to WHO, Indonesia is included in the top 30 countries with high TB/TB-HIV/MDR-TB burden. Indonesia itself has an estimated 6,800 new cases of MDR-TB every year, which comprise $2.8 \%$ of new cases and $16 \%$ of previously treated cases. Managing MDR-TB in Indonesia remains a challenge: surveillance data from 2015 showed that the rate of successful treatment of MDR-TB was only $51 \%$ [1-3]. In addition to its relatively low cure rate, MDR-TB treatment requires regular monitoring of the patient's compliance and multiple adverse drug reactions. These factors account for the high cost of MDR-TB management.

Demographically, Indonesia is comprised of a dense population with rich ethnicities and various culture/tradition-influenced lifestyles. West Papua, a province in the east of Indonesia, has a diverse population that consists of indigenous Papuans and local immigrants. In addition to the mixed ethnicity of West Papua, the region has inadequate health care facilities because it is a distant rural area with underdeveloped infrastructure. These conditions, combined with limited health surveillance data and multiple disease burdens including epidemic TB, mean that treating TB/MDR-TB/TBHIV patients in daily clinical practice is a complex challenge.

The development of drug-resistant TB occurs because of spontaneous mutations among susceptible bacilli; it can be both primary and acquired. Several key risk factors have been identified in patients with MDR-TB, including demographic factors, smoking habits, comorbidities such as HIV and diabetes mellitus (DM), compliance with drug treatment, previous treatment category, strength of positivity of sputum AFB smear, chest radiographic findings, and evidence of extrapulmonary tuberculosis. [5] Two casecontrol studies by Barroso et al. [6] (2003) and Rifat et al. [7] (2014) found that current smoking and a past history of smoking are risk factors for the development of MDR-TB.

Chuchottaworn et al. [8] (2015) reported that being HIV positive was not a risk factor for MDR-TB, although Swaminathan et al. [9] (2005) showed that the proportion of TB drug resistance was higher among HIV-positive patients. These inconsistent findings prompted the investigation of whether being HIV positive is a risk factor, because of the high prevalence of HIV in Indonesia. A recent metaanalysis by Liu et al. [10] (2017) involving 9289 individuals showed that there was a significant association between DM and MDR-TB. Several studies have also shown that other risk factors including treatment failure or discontinuation, sputum AFB smear score+3, and having pleural effusion/cavitation are significantly associated with MDR-TB $[8,11]$.

This case-control study aimed to identify the characteristics of patients and risk factors associated with the development of MDRTB among patients with relapsed TB in West Papua province, Indonesia. The data were obtained indirectly from the medical 
records of patients who had been treated at one of the government hospitals responsible for MDR-TB referral and treatment. The results of this study are expected to provide insight into MDR-TB in West Papua and enhance health prevention and management of MDR-TB in the diverse population of Indonesia.

\section{MATERIALS AND METHODS}

This study was conducted in a referral center of West Papua province, Indonesia, as a retrospective case-control study with ageand sex-matched controls and patients with suspected pulmonary MDR-TB. Data were obtained indirectly from the medical records of patients enrolled between January 2014 and September 2017 at Sorong Regency Regional General Hospital in West Papua. A total of 549 patients suspected to have MDR-TB who registered at the directly observed treatment, short course (DOTS) clinic were included. Inclusion criteria were: (1) $\geq 17$ y old, (2) a history of consumption of anti-TB drugs, (2) Mycobacterium tuberculosis detected by GeneXpert (Xpert MTB/RIFassay) and the Ziehl-Neelsen (acid fast staining) method, and (3) complete data on the TB database. An exclusion criterion was unavailable data for the diagnosis and/or management of previous/present pulmonary TB. (fig. 1)

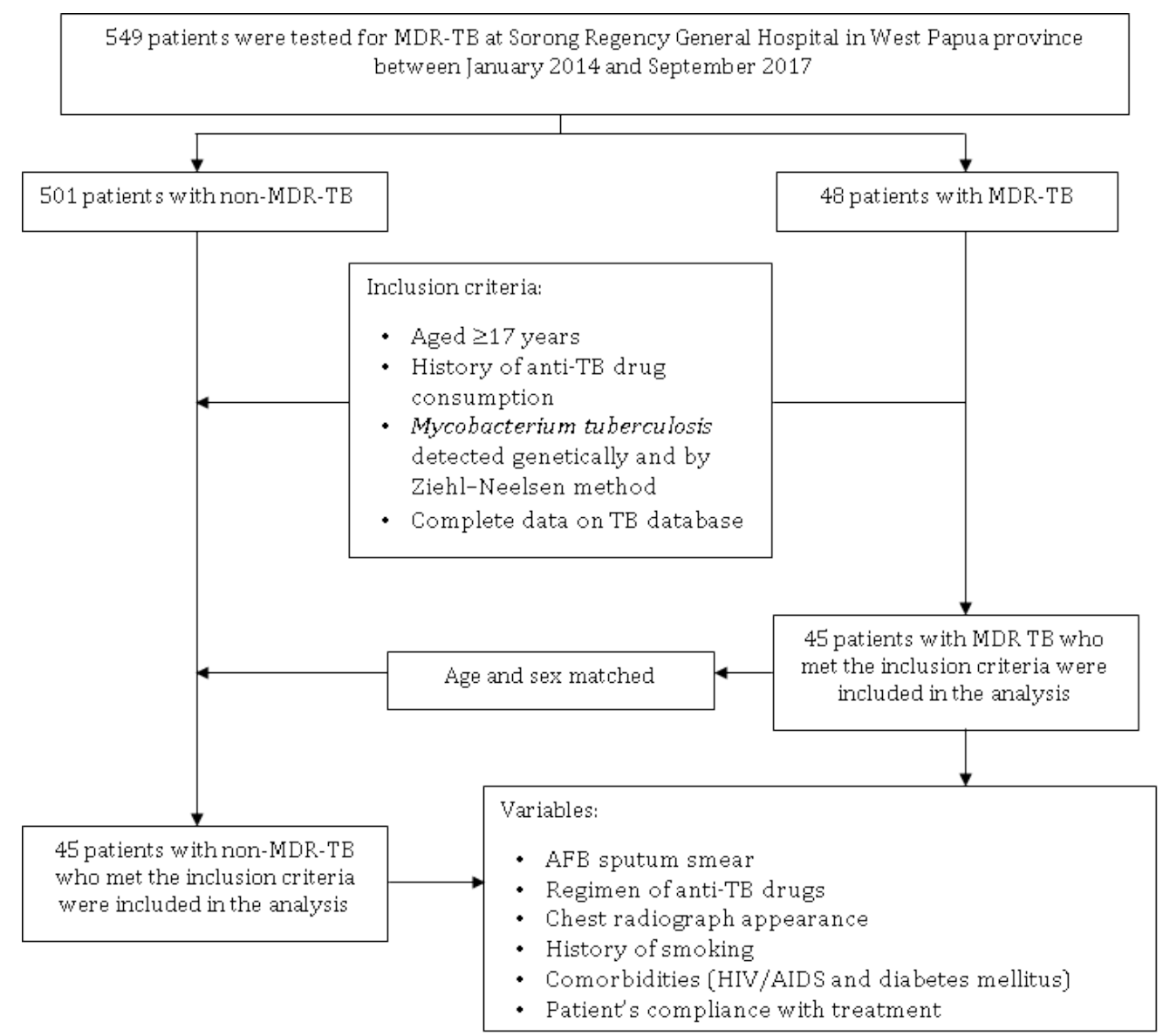

Fig. 1: Scheme of data collection Abbreviation: MDR-TB: multidrug-resistant tuberculosis

Of the patients registered, 48 were diagnosed with MDR-TB; three of these patients were excluded because they had primary drugresistant TB or incomplete data about previous TB medications. The selection of matched controls was randomized among patients with non-MDR TB; the age and sex of MDR-TB subjects were matched with those of 501 non-MDR TB patients who fit the inclusion criteria. Age differences were classified as \pm 3 y. Data were usually acquired from the basic information questionnaire about TB that was completed by the general practitioner and the internal medicine specialist in charge of the DOTS clinic. The patients were anonymized and collection of the data was approved by the hospital authorities or the committee in charge of prevention and management of TB in the hospital.

Data obtained from the participants included demographic characteristics (age, sex, family members with TB under the same roof), and smoking history as lifestyle behavior. The medication history that was obtained from these patients was classified into several categories (lost to follow-up, failure of treatment, completed treatment without follow-up, and relapse), and the number of months patients consumed TB medications was further classified by whether they completed the 2-month intensive phase of treatment. Patients who underwent non-first-line regimens of TB medication or secondcategory treatment were also documented. Supporting examinations such as evidence of extrapulmonary TB, pretreatment AFB sputum smear (strength of positivity), and comorbidities (HIV/AIDS and DM) were documented. The radiology information was the radiologist's interpretation of a plain chest radiograph, the results of which were categorized as presence or absence of infiltrate in the lung field, and the presence of cavitation, pneumothorax, fibrosis, atelectasis, or pleural effusion. TB diagnosis in this study was based on AFB staining of a sputum smear using the Ziehl-Neelsen method and culture resistance was assessed using GeneXpert.

Demographic and clinical data from the participants were analyzed using IBM Corp. released 2012 IBM SPSS Statistics for windows version 21, Armony NY: IBM Group. Descriptive analysis compared the two groups: MDR-TB subjects (case group) vs non-MDR-TB subjects (control group). Further analysis of variables included in this study was performed using the chi-squared test while variable(s) that could not be analyzed with this test were evaluated using Fisher's exact test. 


\section{RESULTS}

This study included 90 participants: 45 patients with MDR-TB (the case group) and 45 patients with non-MDR TB (the control group).
The participants in each group were predominantly men (66.7\%) ( 30 vs 15). The age of the study population ranged from 17 to $78 \mathrm{y}$ with a median of 38 and $40 \mathrm{y}$ for the case and control groups, respectively (table 1).

Table 1: Characteristics of subjects

\begin{tabular}{lll}
\hline Characteristics of the sample & MDR-TB N (\%) & Non-MDR-TB N (\%) \\
\hline Gender & $30(66.6)$ & $30(66.6)$ \\
Male & $15(33.4)$ & $15(33.4)$ \\
Female & & 40 \\
Age & 38 & 17 \\
Median & 18 & 78 \\
Min & 76 & $0(0)$ \\
Max & & $45(100)$ \\
Family history of tuberculosis & $9(20)$ & \\
Yes & $36(80)$ & 0 \\
No & & $45(100)$ \\
Extrapulmonary tuberculosis & $1(2.2)$ & \\
Yes & $44(97.8)$ & $26(57.8)$ \\
No & & $6(13.3)$ \\
Categories of medication history & $20(44.4)$ & $7(15.6)$ \\
Patients lost to follow-up & $7(15.6)$ & $6(13.3)$ \\
Failed to convert & $4(8.9)$ & $14(31.1)$ \\
Complete & & \\
Relapse &
\end{tabular}

Documentation of the social background showed that none of the control group shared a house with a family member with TB or had a confirmed diagnosis of extrapulmonary TB. In contrast, nine subjects from the case group $(20 \%)$ had family who had been diagnosed with TB and one subject (1.1\%) had extrapulmonary TB (table 1).
Comparison of the history of consumption of anti-TB drugs for evaluation of the compliance of the subject population in both groups showed corresponding numbers in each category. Both case and control groups were dominated by subjects that fell within the default/discontinue category: $44.4 \%$ and $57.8 \%$, respectively. However, there was a marked distinction in the category of relapse: $31.1 \%$ in the case group vs $13.3 \%$ in the control group (table 1 ).

Table 2: Medication history (Category I) of subjects

\begin{tabular}{lll}
\hline Duration of Category I medication (months) & MDR-TB N (\%) & Non-MDR-TB N (\%) \\
\hline 1 & $1(2.2)$ & $6(13.3)$ \\
2 & $4(8.9)$ & $7(15.6)$ \\
3 & $10(22.2)$ & $11(24.4)$ \\
4 & $4(8.9)$ & $2(4.4)$ \\
5 & $3(6.7)$ & $2(4.4)$ \\
6 & $20(44.4)$ & $16(35.6)$ \\
7 & $1(2.2)$ & 0 \\
9 & $1(2.2)$ & $1(2,2)$ \\
12 & $1(2.2)$ & 0 \\
\hline
\end{tabular}

Table 2 shows the number of months of first-line anti-TB drug consumption in the case and control groups. Further documentation showed a lack of compliance in reaching the third month of consumption: $33.3 \%$ in the case group and $53.3 \%$ in the control group. However, a significant number of subjects in both the case and control groups ( $44.4 \%$ and $35.6 \%$, respectively) reached their sixth month of therapy, and there were several subjects who consumed anti-TB medication for more than six months $(6.6 \%$ in the case group and $2.2 \%$ in the control group).

A comparison of the risk factors between the case and control groups included smoking habits, chest radiographic appearance, status and duration of previous TB treatment, comorbidities (HIV and DM), category of previous anti-TB drug consumption, and sputum culture results.

The proportion of smokers in both groups was similar. Patients who were current or past regular smokers comprised $53.3 \%$ of the case group and $55.6 \%$ of the control group. Statistical analysis found no significant association of this factor with MDR-TB vs non-MDR-TB (P = 0.399).
All patients included in this study were tested for HIV. The proportion of HIV-positive patients was higher in the case group than in the control group (6 [13.3\%] vs 2 [4.4\%] patients), but this difference was not significant. Similar results were found for chest radiographic interpretations and history of non-first-line TB treatment. The case group had a higher number of subjects with noninfiltrated or typical radiological images compared with the control group (7 [15.6\%] vs 3 [6.7\%]; $\mathrm{P}=0.180$ ).

However, variable chest radiograph interpretations were found in the case group (table 3): three patients had lung cavitation, three had atelectasis, and one patient had a fibrotic lung. Statistical analysis of the history of non-first-line TB treatment in both groups showed no significant difference, despite a higher proportion in the case group compared with the control group (11 [24.4\%] vs 6 [13.3\%] subjects, respectively; $\mathrm{P}=0.178$ ). In contrast, in the control group, a greater number of subjects had failed or defaulted previous TB treatment compared with the case group (32 [71.1\%] vs 27 [60\%] subjects; $\mathrm{P}=0.267$ ) (table 4). 
Table 3: Bivariate analysis of variables

\begin{tabular}{|c|c|c|c|c|}
\hline Variables & MDR-TB N (\%) & Non-MDR TB N (\%) & P-value & OR (95\% CI) \\
\hline \multicolumn{5}{|l|}{ Smoking status } \\
\hline Yes & $21(53.3)$ & $25(55.6)$ & 0.399 & $0.71(0.3-1.6)$ \\
\hline No & $24(46.7)$ & $20(44.4)$ & & \\
\hline \multicolumn{5}{|l|}{ HIV } \\
\hline Positive & $6(13.3)$ & $2(4.4)$ & 0.138 & $3.3(0.63-17.36)$ \\
\hline Negative & $39(86.7)$ & $43(95.6)$ & & \\
\hline \multicolumn{5}{|l|}{ Diabetes mellitus } \\
\hline Yes & $10(22.2)$ & $2(4.4)$ & $0.013^{*}$ & $6.14(1.26-29.89)$ \\
\hline No & $35(77.8)$ & $43(95.6)$ & & \\
\hline \multicolumn{5}{|l|}{ Thoracic X-ray } \\
\hline Infiltrate & $38(84.4)$ & $42(93.3)$ & 0.180 & $2.579(0.62-10.69)$ \\
\hline No infiltrate & $7(15.6)$ & $3(6.7)$ & & \\
\hline \multicolumn{5}{|l|}{ AFB sputum smear } \\
\hline+3 & $18(40)$ & $5(11.1)$ & $0.002^{*}$ & 5.33 (1.76-16.09) \\
\hline$<+3$ & $27(60)$ & $40(88.9)$ & & \\
\hline \multicolumn{5}{|l|}{ Duration of category I } \\
\hline Completed intensive phase & $40(88.9)$ & $32(71.1)$ & $0.035^{*}$ & $3.25(1.04-10.07)$ \\
\hline Not completed intensive phase & $5(11.1)$ & $13(28.9)$ & & \\
\hline \multicolumn{5}{|l|}{ Treatment status } \\
\hline Default or failure & $27(60)$ & $32(71.11)$ & 0.267 & $0.609(0.253-1.466)$ \\
\hline Other & $18(40)$ & $13(28,89)$ & & \\
\hline \multicolumn{5}{|l|}{ Category II treatment } \\
\hline Yes & $11(24.4)$ & $6(13.33)$ & 0.178 & $2.103(0.703-6.292)$ \\
\hline Non-category II & $34(75.56)$ & $39(86.67)$ & & \\
\hline
\end{tabular}

$* \mathrm{P}<0.05$

Table 1: Chest radiograph appearance

\begin{tabular}{lll}
\hline & MDR-TB & Non-MDR-TB \\
\hline Thoracic X-ray appearance & 3 cavities & 2 pleural effusion \\
& 3 atelectasis & 1 pneumothorax \\
& 1 fibrotic lung & \\
\hline
\end{tabular}

DM was identified in 10 patients $(22.2 \%)$ in the case group and two patients $(4.4 \%)$ in the control group. Further statistical analysis found a significant association of this risk factor with the development of MDR-TB (OR: 6.14, 95\% CI 1.26-29.89; P = 0.013). In the case group, significantly more patients had completed the intensive phase of firstline anti-TB treatment ( $2 \mathrm{mo}$ ) compared with the control group (40 [88.9\%] vs 32 [71.1\%]; OR: 3.25, 95\% CI 1.04-10.07; $\mathrm{P}=0.035$ ). Staining for AFB in sputum of patients included in this study found that $18(40 \%)$ in the case group vs only five $(11.1 \%)$ in the control group had a result of +3 . Further analysis found that an AFB score of +3 was significantly associated with MDR-TB compared with a score of less than+3 (OR: 5.33, 95\% CI 1.76-16.09; $\mathrm{P}=0.002$ ).

\section{DISCUSSION}

This study provides the first description of TB patients, especially those with MDR-TB, in the region of West Papua, Indonesia. Regarding the prevalence of MDR-TB in the region, we report the first data confirming the occurrence of drug resistance among patients with relapsed TB. The study was conducted among relapsed patients because of previous evidence showing that patients with a history of TB treatment have an estimated 10 times higher risk of developing MDR-TB.

In our study, most patients with MDR-TB were male (66.6\%), and a family history of tuberculosis was only identified in $20 \%$ of the case group. This gender proportion was similar to that in studies by Magee et al.[5] and Chuchottaworn et al.[8], where men dominated the patients with MDR. The present study did not analyze gender as a risk factor, although Faustini et al.[12] hypothesized that factors such as compliance might have played a role in this phenomenon. Although contact with TB patients has often been reported as a risk factor for primary TB, there is limited information about the family history of TB for patients with MDR-TB. The number of cases in the present study is similar to that in Casal et al.[13] and Raazi et al.,[14] who found that respectively $22.5 \%$ and $20.37 \%$ of patients with MDR-TB had a family history of TB. However, neither of these studies found a significant association of this variable with MDR-TB.
Among the relapsed patients who were included in the present study, the majority of both groups fell within the category of lost to follow-up after previous treatment. Further analysis comparing the medication status of category I treatment found that it was not a significant risk factor for the development of MDR-TB, although completing the intensive phase ( $2 \mathrm{mo}$ ) of category I treatment was found to be associated with the occurrence of MDR-TB. Analysis of these data should be critically addressed. A study by Hirpa et al. found similar results, in that patients who underwent 2-7 mo of treatment were classified as at risk of MDR-TB.[15] Cox et al. studied the biomolecular changes that amplify resistance and found these to be highest during the intensive phase of treatment when patients were taking the most drugs and/or were hospitalized. This study also suggested the possibility that patients were infected with multiple strains of TB, and after anti-TB drugs were administered, the resistant strain remained whereas drug-susceptible strains were diminished.[16] Another factor that should be considered when addressing this finding is the low compliance among TB patients in the maintenance phase of treatment, as reported by Castelnuovo.[17] However, the end result of category I treatment is not consistent with previous evidence reported by Stosic et al., namely that treatment default and loss to follow-up are risk factors for MDR-TB.[18]

In the present study, comorbidities such as HIV and DM were found to be more prevalent in the case group compared with the control group, but statistical analysis of these comorbidities found that only DM was significant. A meta-analysis including 9289 patients from a number of studies found that DM increased the risk of TB (pooled statistic OR: 1.71, 95\% CI 1.32-2.22).[10] This finding is supported by previous study who reported that DM patients with TB usually had higher mycobacterial loads and therefore a higher possibility of bacillary mutation and the occurrence of MDR. They also noted that patients with DM had impaired immune responses marked by decreased production of immune components such as interferon- $\gamma$. In addition, Pasipanodya et al. found that patients with DM had 53\% 
lower plasma levels of rifampicin.[19] Despite the lack of significance of the association of HIV and MDR-TB in the present study, the results show a trend toward association compared with drug-sensitive $\mathrm{TB}$, which is consistent with previous reports.

Our study showed that patients who had a positive sputum AFB smear score of +3 had a higher risk of developing MDR-TB. This finding accords with that of Chuchottaworn et al., who also demonstrated an association of sputum AFB smear score +3 with MDR-TB (OR: 13.09, 95\% CI 4.64-36.91).[8] Caetano Mota et al.[20] found that patients with a sputum AFB smear score of +3 had an 11.7 times higher risk of delayed sputum conversion because of the high bacterial density in sputum. This might affect the development of drug-resistant strains of TB during treatment. Djouma et al. also reported that a pretreatment sputum AFB score of +3 was associated with a higher rate of treatment failure and delayed sputum conversion [21].

In contrast to other studies, our study found that smoking history was not a risk factor for developing MDR-TB. This finding is probably because of the prevalence of smoking in both case and control groups: over $50 \%$ of the relapsed patients had a history of smoking. This result should be acknowledged because it has been shown that smoking is one of the determinants of TB, and might be the predictor of a delayed response to anti-TB drugs [5].

The results of interpretation of the chest radiographs showed that MDR-TB (case group) patients had a slightly different appearance compared with those with non-MDR TB (control group). Variant chest radiographic findings including lung cavitation, atelectasis, and fibrotic lung were identified in the case group. Although the differences between groups were not statistically significant, they might affect the prognosis and morbidity of the patients. Two studies have reported the presence of lung cavitation as a risk factor for MDR-TB $[6,11]$. Although the literature concerning the relationship between lung cavitation and MDR-TB is scarce, it could be hypothesized that the conditions inside the cavitations (high level of oxygenation and protection by the cavity wall) could prevent the anti-TB drugs reaching adequate inhibitory concentrations.

This study was conducted in a rural area of Indonesia where health education, including that about communicable diseases like TB and HIV, is limited. This study faced several limitations in the collection of data because registration and data availability are not fully computerized and recorded. The present study also had a limited sample size for several reasons: for instance, there were a number of subjects who resided in more distant areas where limited access to transportation might create obstacles to health access. Thus, limited data were available for these subjects.

However, the present study provides the first view of MDR-TB patients in West Papua province, where Sorong Regency Regional Hospital has become the referral center for MDR-TB patients. In addition to the geographical obstacles that the region faces, it has limited numbers of health centers that can provide treatment for TB patients, especially those with MDR-TB.

\section{CONCLUSION}

This study identified a number of relapsed patients who were diagnosed with MDR-TB. Statistical analysis of this population identified some alarming information; for instance, only the MDR-TB case group contained patients who had extrapulmonary TB or a family history of TB, and it contained a larger proportion of patients with HIV. Further analysis showed that DM, previous completion of 2 mo of intensive TB treatment, and a score of +3 for sputum AFB were associated with MDR-TB.

\section{ACKNOWLEDGMENT}

This article was presented at The $3^{\text {rd }}$ International Conference and Exhibition on Indonesian Medical Education and Research Institute (ICE on IMERI 2018), Faculty of Medicine, Universitas Indonesia, Jakarta, Indonesia. We acknowledge the support of the hospital board of Sorong Regency Regional Hospital, West Papua, Indonesia and colleagues from the medical team including doctors, nurses, and operational staff in the DOTS clinic of the hospital. We thank the 3rd
ICE on IMERI Committee who had supported the peer review and manuscript preparation before submitting to the journal.

\section{AUTHORS CONTRIBUTIONS}

All the author have contributed equally

\section{CONFLICT OF INTERESTS}

The author reports no conflicts of interest in this work. This study received no funding from an appropriate or relevant institution.

\section{REFERENCES}

1. World Health Organization. Global Tuberculosis Report; 2017. p. 16-61.

2. WHO Indonesia Tuberculosis Profile; 2017. Available from: https://extranet.who.int/sree/Reports?op=Repletandname $=\%$ 2FWHO_HQ_Reports\%2FG2\%2FPROD\%2FEXT\%2FTBCountry ProfileandISO2=IDandLAN=ENandouttype=pdf [Last accessed on 18 Oct 2018]

3. USAID. Indonesia MDR-TB country profile; 2016. Available from:

https://www.usaid.gov/sites/default/files/documents/1864/I ndonesia_MDR-TB_508_ck.pdf [Last accessed on 18 Oct 2018]

4. WHO. Multidrug resistant tuberculosis (MDR-TB); 2017. Available http://www.who.int/tb/challenges/mdr/MDR-

RR_TB_factsheet_2017.pdf [Last accessed on 18 Oct 2018]

5. Magee MJ, Kempker RR, Kipiani M. Diabetes mellitus, smoking status, and rate of sputum culture conversion in patients with multidrug-resistant tuberculosis: a cohort study from the country of Georgia. PLoS ONE 2014;9:1-9.

6. Barroso EC, Mota RMS, Santos RO, Sousa ALO, Barroso JB, Rodrigues JLN. Risk factors for acquired multidrug-resistant tuberculosis. J Pneumol 2003;29:89-97.

7. Rifat M, Milton AH, Hall J. Development of multidrug resistant tuberculosis in Bangladesh: a case-control study on risk factors. PLOS ONE 2014;9:2-8.

8. Chuchottaworn C, Thanachartwet V, Sangsayunh P. Risk factors for multidrug-resistant tuberculosis among patients with pulmonary tuberculosis at the central chest institute of Thailand. PLoS ONE 2015;10:1-17.

9. Swaminathan S, Paramasivan CN, Ponnuraja C, Iliayas S, Rajasekaran S, Narayanan PR. Anti-tuberculosis drug resistance in patients with HIV and tuberculosis in South India. Int J Tuberc Lung Dis 2005;9:896-900.

10. Liu Q, Li W, Xue M. Diabetes mellitus and the risk of multidrug resistant tuberculosis: a meta-analysis. Sci Rep 2017;7:1-7.

11. Mulu W, Mekonnen D, Yimer M, Admassu A, Abera B. Risk factors for multidrug resistant tuberculosis patients in Amhara National Regional State. Afr Health Sci 2015;15:368-77.

12. Faustini A, Hall AJ, Perucci CA. Risk factors for multidrug resistant tuberculosis in Europe: a systematic review. Thorax 2006;61:158-63.

13. Casal M, Vaquero M, Rinder H. A case-control study for multidrug-resistant tuberculosis: Risk factors in four European countries. Microb Drug Resist 2005;11:62-7.

14. Raazi J, Prakash S, Parveen K, Shaikh S. Risk factors of multidrug resistant tuberculosis in urban Allahabad, India. Int J Community Med Public Health 2017;4:2383-8.

15. Hirpa S, Medhin G, Girma B. Determinants of multidrugresistant tuberculosis in patients who underwent first-line treatment in addis ababa: a case control study. BMC Public Health 2013;13:1.

16. Cox HS, Niemann S, Ismailov G. Risk of acquired drug resistance during short-course directly observed treatment of tuberculosis in an area with high levels of drug resistance. Clin Infect Dis 2007;44:1421-7.

17. Castelnuovo B. A review of compliance to anti tuberculosis treatment and risk factors for defaulting treatment in Sub Saharan Africa. Afr Health Sci 2010;10:320-4.

18. Stosic M, Vukocic D, Babic D, Antonijevic G, Foley KL, Vujcic I, et al. Risk factors for multidrug-resistant tuberculosis among tuberculosis patients in Serbia: a case-control study. BMC Public Health 2018;18:1114. 
19. Pasipanodya JG, Srivastava S, Gumbo T. Meta-analysis of clinical studies supports the pharmacokinetic variability hypothesis for acquired drug resistance and failure of antituberculosis therapy. Clin Infect Dis 2012;55:169-77.

20. Caetano Mota P, Carvalho A, Valente I, Braga R, Duarte R. Predictors of delayed sputum smear and culture conversion among a portuguese population with pulmonary tuberculosis. Rev Port Pneumol 2012;18:72-9.

21. Djouma FN, Noubom M, Ateudjieu J, Donfack H. Delay in sputum smear conversion and outcomes of smear-positive tuberculosis patients: a retrospective cohort study in Bafoussam, Cameroon. BMC Infect Dis 2015;15:1-7. 